\title{
Application of R-Functions Method and Parallel Computations to the Solution of 2D Elliptic Boundary Value Problems
}

\author{
Marcin Detka and Czesław Cichoń \\ Chair of Applied Computer Science, Kielce University of Technology, \\ Al. Tysiąclecia Państwa Polskiego 7, \\ 25-314 Kielce, Poland \\ \{Marcin.Detka, Czeslaw.Cichon\}@tu.kielce.pl
}

\begin{abstract}
In the paper, the R-function theory developed by Rvachew is applied to solve 2D elliptic boundary value problems. Unlike the well-established FEM or BEM method, this method requires dividing the solution into two parts. In the first part, the boundary conditions are satisfied exactly and in the second part, the differential equation is satisfied in an approximate way. In such a way, it is possible to formulate in one algorithm the so-called general structural solution of a boundary-value problem and use it for an arbitrary domain and arbitrary boundary conditions. The usefulness of the proposed computational method is verified using the example of the solution of the Laplace equation with mixed boundary conditions.
\end{abstract}

Keywords: structural solution, R-functions, parallel computations.

\section{Introduction}

Mathematical models of engineering problems are often defined as boundary-value problems involving partial-differential equations. For the description of such problems it is required to have analytical information connected with the equation itself (or a set of equations) and geometrical information necessary to define boundary conditions. This information concerns the solution domain, shapes of particular parts of the boundary, distribution and forms of the imposed constraints and the like. It is accounted for in a different way in various solution methods. In the paper, such problems are solved in a uniform way using the R-function theory, developed by Rvachew et al. [3]. In this theory, the so-called structural solutions are constructed with the use of elaborated tools of the analytical geometry. As a result, the structural solution exactly satisfying the boundary conditions contains some unknown parameters that have to be computed.

The paper is limited to elliptic problems in two dimensions. Such problems are still dealt with because of their well-known relation to many physical models. Furthermore, theoretical and numerical results obtained in this area are very useful in practice. 
The discrete solution is determined using orthogonal, structured grid nodes over the Cartesian space $C$ which contains the solution domain $\Omega$. The unknown function of the problem is approximated by means of assuming a set of simple spline functions of the first order. The property of the support locality and density of these functions make it possible to compute, in an effective way, parameters of the structural solution, by redistributing the solution procedure into processors. In the algorithm of the parallel solution, the meshless method, proposed by Yagawa et al. [6], is applied. In this method, the resulting system of linear equations is constructed in a "row-by-row" fashion.

The usefulness of the proposed method of computations is verified with the example of the solution of the Laplace equation with mixed boundary conditions.

\section{Problem Statement and the Method of Solution}

Consider the linear operator equation of the form:

$$
A u=f \quad \text { in } \quad \Omega \subset \mathfrak{R}^{2},
$$

where $f \in L_{2}(\Omega)$.

It is well known, that when $A$ is a linear positive-defined operator on a linear set $\mathrm{D}_{\mathrm{A}}$ in a separable Hilbert space $H$, and $f \in H$, the generalized solution $u$ of Eq. (1) is an element of the so-called energy space $H_{\mathrm{A}}$ that minimizes the functional [2]:

$$
J(u)=\frac{1}{2} B(u, u)-(f, u)_{H},
$$

where $B(u, u)=(A u, u)_{H}$ and $(f, u)_{H}$ are bilinear and linear functionals, respectively.

Because of the equivalence of Eqs. (1) and (2), in numerical computations it is preferred to solve Eq. (2) using the Ritz method. It is assumed that for the most general boundary conditions the solution can by represented in the structural form:

$$
u=\phi_{0}+\omega \varphi\left(\phi_{1}\right)
$$

where $\omega$ is a known function that takes on zero values on the boundary $\partial \Omega$ and is positive in the interior of $\Omega$. Functions $\phi_{0}$ and $\phi_{1}$ are chosen in such a way so as to satisfy all boundary conditions. The specification of the function $\varphi$ depends on the problem under consideration (see Section 4). It should be noted that functions $\phi_{0}$ and $\phi_{1}$ can by specified in a piece-wise fashion with different values prescribed to them at each part of the boundary $\partial \Omega$. The advantage of the solutions in the form of Eq. (3) 
is that the function $\omega$ describes completely all the geometrical information of a particular boundary value problem. The equation $\omega=0$ defines the geometry of a domain implicitly. The functions $\omega$ are constructed using the theory of R-functions developed by Rvachev [3].

Finally, functions $\phi_{0}$ and $\phi_{1}$ can by expressed by only one function $\phi$ [5] that in the Ritz approximation is sought in the form:

$$
\phi_{N}=\sum_{j=1}^{n} c_{j} \psi_{j}
$$

where $N$ is a positive integer, $c_{j}$ are unknown parameters and $\left\{\psi_{j}\right\}$ are some basis functions. The sole purpose of the function $\phi_{N}$ is to satisfy the analytical constraints of the boundary value problem. It means that the structure of Eq. (3) does not place any constraints on the choice of the functions $\psi_{j}[4]$

After integrating over the domain $\Omega$, the functional $J(u)$ becomes an ordinary function of the parameters $c_{1}, c_{2}, \ldots, c_{N}$. Therefore, the condition $\delta J=0$ is equivalent to the solution of the linear algebraic equation, characterized by the matrix equation:

$$
\mathbf{K c}=\mathbf{F} .
$$

\section{Parallel Procedure of Computations}

Basis functions $\left\{\psi_{j}\right\}$ can be defined globally in the domain $\Omega$, or locally with the dense local supports. As regards the parallel solution of the problem, the local approach is preferable, therefore it is chosen in the paper.

Let us define the Cartesian space $C \subset \mathfrak{R}^{2}$ and assume that the solution domain is subspace $\Omega \subset C$, Fig. 1 . Then, the space $C$ is discretized using the regular mesh points of the structured grid. It is necessary to choose integers $n$ and $m$, and define step size $h$ and $k$ by $h=\left(x_{b}-x_{a}\right) / n$ and $k=\left(y_{b}-y_{a}\right) / m$, where points $a\left(x_{a}, y_{a}\right)$ and $b\left(x_{b}, y_{b}\right)$ are given a priori. For each point of the grid, the simple spline of the first order based on the sixth triangles, containing the grid vertex $j$ is defined, Fig. 2. The basis function $\psi_{j}$ is composed of the six linear functions:

$$
\psi_{j}=\left\{\psi_{j}^{1}, \psi_{j}^{2}, \ldots, \psi_{j}^{6}\right\}
$$

where functions $\psi_{j}^{k}, k=1,2, \ldots, 6$, have the following form in the local coordinate system $\left(s_{1}=\left(x-x_{j}\right) / h, s_{2}=\left(y-y_{j}\right) / k\right)$ : 


$$
\psi_{j}=\left\{\begin{array}{ll}
\psi_{j}^{1}=1-s_{1}-s_{2} & \text { if }\left(s_{1}, s_{2}\right) \in T_{1} \\
\psi_{j}^{2}=1-s_{2} & \text { if }\left(s_{1}, s_{2}\right) \in T_{2} \\
\psi_{j}^{3}=1+s_{1} & \text { if }\left(s_{1}, s_{2}\right) \in T_{3} \\
\psi_{j}^{4}=1+s_{1}+s_{2} & \text { if }\left(s_{1}, s_{2}\right) \in T_{4} \\
\psi_{j}^{5}=1+s_{2} & \text { if }\left(s_{1}, s_{2}\right) \in T_{5} \\
\psi_{j}^{6}=1-s_{1} & \text { if }\left(s_{1}, s_{2}\right) \in T_{6} \\
0 & \text { otherwise }
\end{array} .\right.
$$

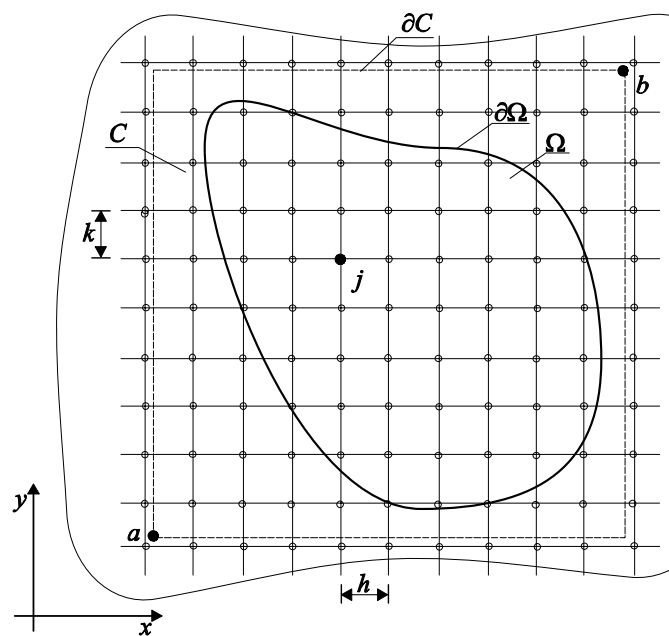

Fig. 1. Cartesian space $\mathrm{C}$ and the solution domain $\Omega$
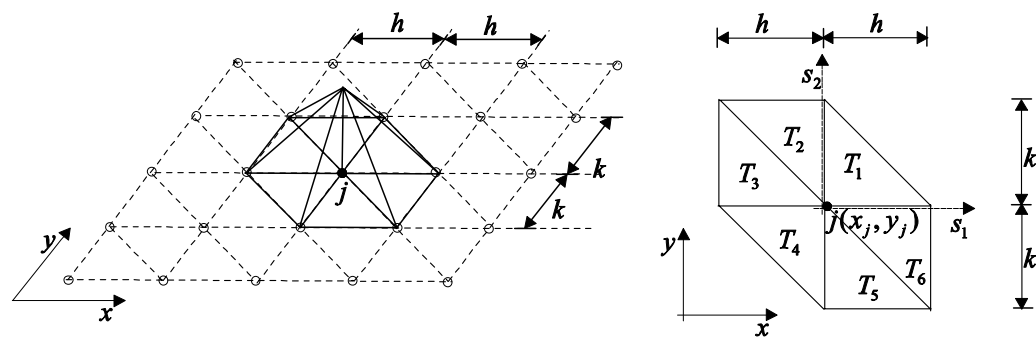

Fig. 2. Linear dashed basis function $\psi_{j}$ 
The algorithm of parallel computations is shown in Fig. 3. The main steps of the computations are as follows:

1. Decomposition of the space $C$ into $C_{p}$ subdomains, $p=1,2, \ldots, P$, where $P$ is the number of processors.

2. Parallel identification of nodes in each subdomain $C_{p}$ according to the rule shown in. Fig. 4.

3. Parallel modification of subdomain $C_{p}$ in order to balance the number of nodes in each processor.

4. Parallel supplement of the nodes set in the domain $C_{p}$ with neighbouring nodes which are active in the solution of the problem.

5. For each node $\mathrm{j}$, parallel computations of the elements $K_{j k}$ and $\mathrm{F}_{\mathrm{j}}, k=1,2, \ldots, 7$ (max) of the matrix equation (5).

6. Parallel solution of the matrix equation (5) by the conjugate gradient method using the Portable Extensible Toolkit for Scientific Computation (PETSc) library.

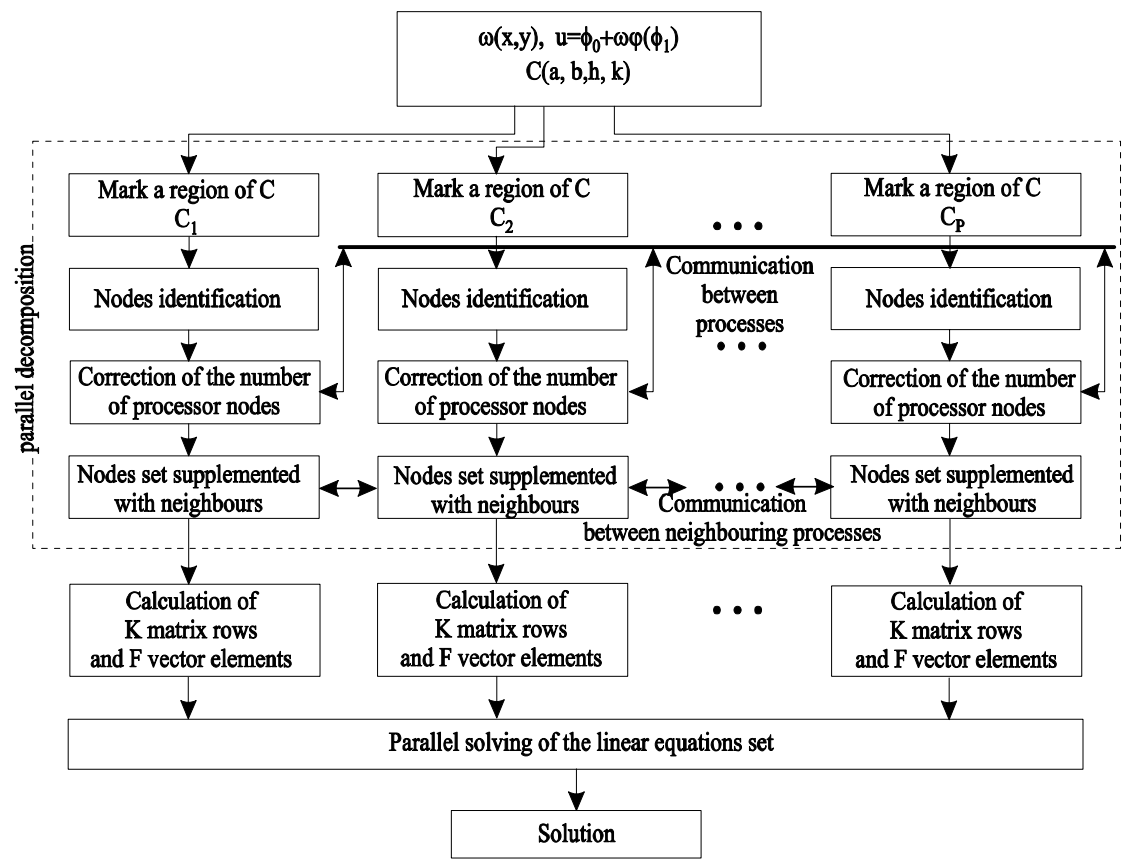

Fig. 3. The algorithm of parallel computations

Numerical integration is needed to calculate the matrix $\mathbf{K}$ and the vector $\mathbf{F}$. Integration over triangles is performed with the use of 4-point Gaussian quadrature. For the case when the boundary $\partial \Omega$ crosses the triangle an additional procedure has been applied in order to divide the integration region into subregions. The rule states 


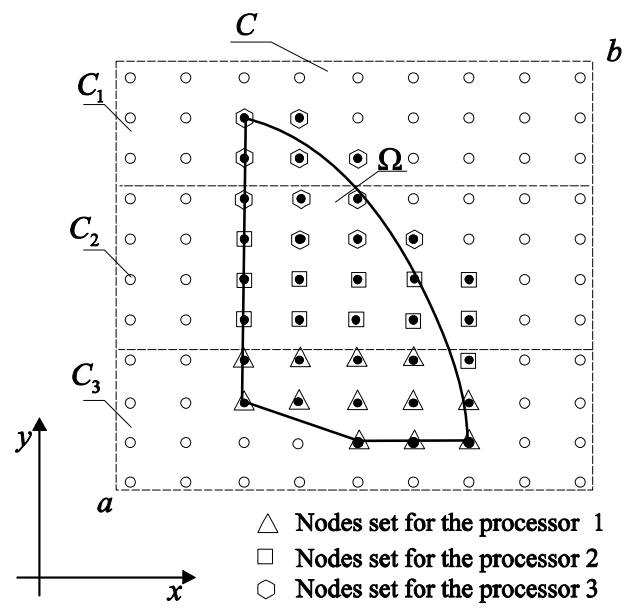

Fig. 4. Decomposition of the solution domain $(\mathrm{P}=3)$, identification of nodes

that "the active subregion" is such a part of the triangle that belongs to the $\Omega$ domain and contains any of integration points. Next, integrals over the new triangle or quadrilateral subregions are also computed numerically. The above rule has also been applied to the identification of nodes in the subdomains.

\section{Example}

The proposed solution method has been verified with a simple example taken from [1].

Consider the Laplace equation on the domain $\Omega$, shown in Fig. 5

$$
-\nabla^{2} u(x, y)=0 \text { in } \Omega,
$$

with the boundary conditions on $\partial \Omega$

$$
\begin{aligned}
& \left.\frac{\partial u}{\partial y}\right|_{\partial \Omega_{1}}=\left.0 \quad \frac{\partial u}{\partial x}\right|_{\partial \Omega_{3}}=-\left.2 \quad u(x, y)\right|_{\partial \Omega_{4}}=80 \\
& \left.u(x, y)\right|_{\partial \Omega_{2}}=-4 x^{4}+33 x^{2}-2 x+17 .
\end{aligned} .
$$

The exact solution is equal to:

$$
u(x, y)=81-2 x+x^{2}-y^{2} .
$$

The geometric domain $\Omega$ can be defined as a Boolean set combination of four primitives

$$
\Omega=\Omega_{1} \cap \Omega_{2} \cap \Omega_{3} \cap \Omega_{4},
$$




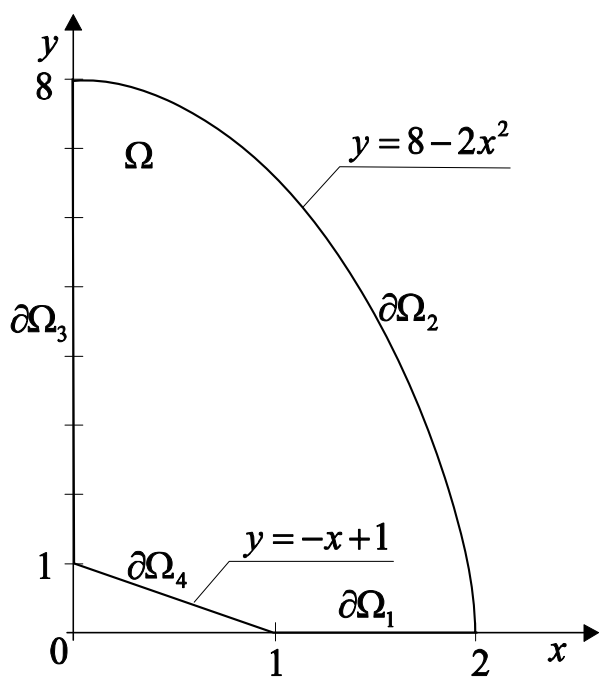

Fig. 5. Solution domain $\Omega$ of the Laplace equation (8)

defined as

$$
\Omega_{i}=\left\{(x, y) \in \mathfrak{R}^{2}: \omega_{i}(x, y) \geq 0\right\}
$$

Functions $\omega_{i}$, normalized to the first order, have the form:

$$
\begin{aligned}
& \omega_{1}=y \quad, \quad \omega_{2}=\frac{8-2 x^{2}-y}{\sqrt{16 x^{2}+1}}, \\
& \omega_{3}=x \quad, \quad \omega_{4}=\frac{\sqrt{2}}{2}(y-1+x),
\end{aligned}
$$

and the equation of the solution domain $\Omega$ can be expressed in the following way:

$$
\omega=\omega_{1} \wedge_{0} \omega_{2} \wedge_{0} \omega_{3} \wedge_{0} \omega_{4}
$$

where $\wedge_{0}$ is the $R_{0}$ - conjunction.

After some manipulations, the structural form of the solution (3) takes the final form

$$
u=g_{01}-\omega D_{1}\left(g_{01}\right)+\omega g_{11}-\omega D_{1}\left(\phi g_{02}\right)+\phi g_{02},
$$

where $D_{1}(\bullet)=\frac{\partial \omega}{\partial x} \frac{\partial(\bullet)}{\partial x}+\frac{\partial \omega}{\partial y} \frac{\partial(\bullet)}{\partial y}$ and 


$$
\begin{aligned}
& g_{01}=\frac{\left(-4 x^{4}+33 x^{2}-2 x+17\right) \omega_{134}+80 \omega_{123}}{\omega_{234}+\omega_{134}+\omega_{124}+\omega_{123}}, g_{11}=\frac{-2 \omega_{1}}{\omega_{1}+\omega_{3}}, \\
& g_{02}=\frac{\omega_{234}+\omega_{124}}{\omega_{234}+\omega_{134}+\omega_{124}+\omega_{123}},
\end{aligned}
$$

where $\omega_{i j k}=\omega_{i} \omega_{j} \omega_{k}$. The functional (2) takes the form

$$
J(u)=\int_{\Omega}\left[\left(\frac{\partial u}{\partial x}\right)^{2}+\left(\frac{\partial u}{\partial y}\right)^{2}\right] d \Omega+4 \int_{\partial \Omega_{3}} u d \partial \Omega_{3} .
$$

The formulae for the calculation of the matrix $\mathbf{K}$ coefficients and the column vector F , Eq. (5), are given explicitly in [1].

Computations have been made for $h=k=0.5,0.2$ and 0.1 , which has led to the sets of the basis functions $\left\{\psi_{j}\right\}, j=1,2,3, \ldots, N$, where $N=63,307$ and 1124 . The quality of the solution has been verified calculating the absolute, relative and least square errors:

$$
\begin{aligned}
& \varepsilon_{1}=\max _{i}\left|u^{\text {exac }}-u^{\text {approx }}\right|, \\
& \mathcal{E}_{2}=\max _{i}\left|\frac{u^{\text {exac }}-u^{\text {approx }}}{u^{\text {exac }}}\right|, \\
& \varepsilon_{3}=\frac{1}{N} \sum_{i}\left(u^{\text {exac }}-u^{\text {approx }}\right)^{2} .
\end{aligned}
$$

The results of computations are given in Table 1. It should be noted that the improvement in the calculation accuracy at higher mesh density is smaller than expected. Probably, the reason is that the basic functions $\psi_{j}$ are too simple. The last column in Table 1 presents the data given in [1], where the global approximations are assumed in the form of the third degree complete polynomial. It should be stressed that although the final solution is worse, it is obtained with notably less numerical effort.

Table 1. Approximation errors

\begin{tabular}{|c|c|c|c|c|}
\cline { 2 - 5 } \multicolumn{1}{c|}{} & $\mathrm{h}=\mathrm{k}=0.5$ & $\mathrm{~h}=\mathrm{k}=0.2$ & $\mathrm{~h}=\mathrm{k}=0.1$ & {$[1]$} \\
\hline$\varepsilon_{1}$ & 6.00 & 2.34 & 1.98 & 10.32 \\
\hline$\varepsilon_{2}$ & 0.15 & 0.05 & 0,04 & 0.41 \\
\hline$\varepsilon_{3}$ & 2.62 & 0.93 & 0.85 & 19.18 \\
\hline
\end{tabular}


The graphs of the $u$ function for different vertical and horizontal cross-sections of the $\Omega$ domain are shown in Fig. 6 .
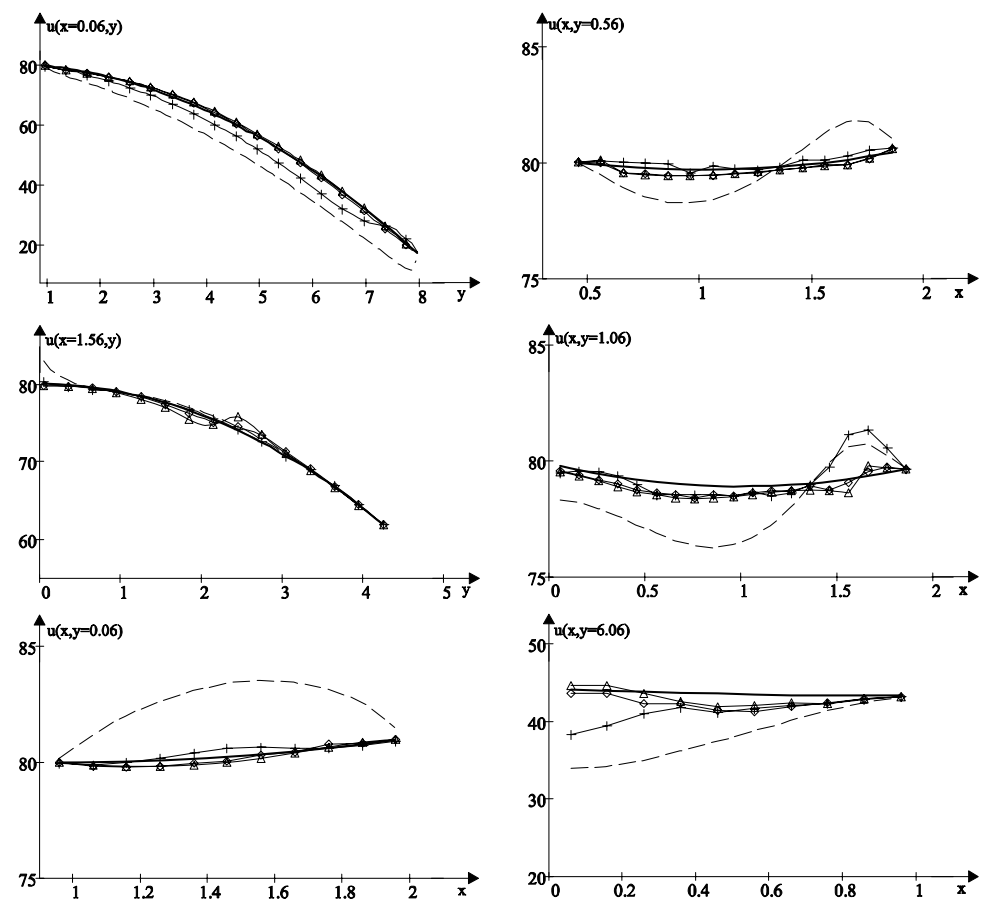

Fig. 6. Graphs of the $u$ function for the different cross-sections, +++ discretization $\mathrm{k}=\mathrm{h}=0.5$, $\Delta \Delta$ discretization $\mathrm{k}=\mathrm{h}=0.2, \Delta \wedge \wedge$ discretization $\mathrm{k}=\mathrm{h}=0.1,---$ polynomial $\mathrm{N}=3$ [1], — exact solution
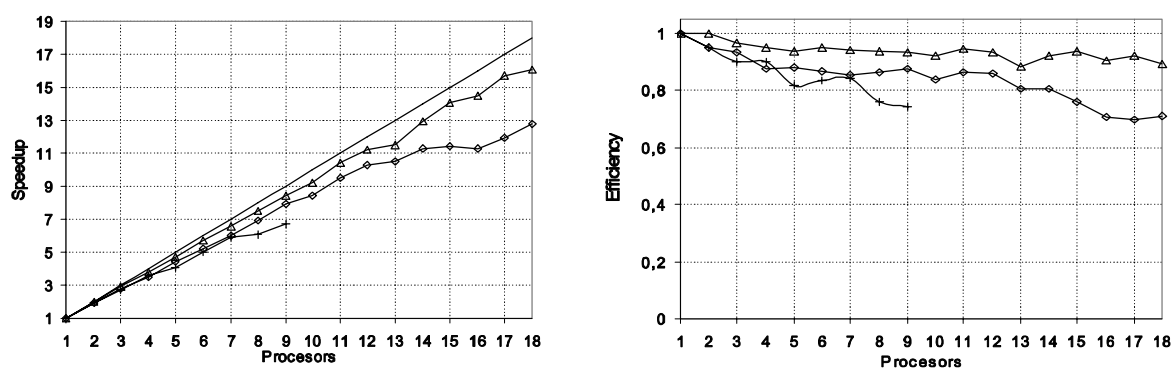

Fig. 7. Speedup and parallel efficiency in the function of the number of processors (time of the parallel solving of the linear equations set has been omitted), - theoretical ideal speedup, +++ discretization $\mathrm{k}=\mathrm{h}=0.5$, $\diamond \diamond \diamond$ discretization $\mathrm{k}=\mathrm{h}=0.2, \Delta \Delta \Delta$ discretization $\mathrm{k}=\mathrm{h}=0.1$ 
As expected, the assumption of simple linear basis functions yields quite satisfactory computational results for suitably dense mesh nodes. Some inaccuracies that occur in the interior of the domain solution probably result from approximate calculations of the function derivatives, which appear in the formulae. In the program, these derivatives are calculated using of the GNU Scientific Library (GSL). Fig. 7 shows how the speedup and parallel efficiency varies with the numbers of processors for various problem sizes. The presented algorithm has been parallelized using Message Passing Interface (MPI MPICH ver. 1.2.7p1) library function and GNU C Compiler (ver.3.2). It has been tested with 9 nodes cluster with 2 Intel Xenon $2.4 \mathrm{Mhz}$ $1 \mathrm{~GB}$ of RAM. The nodes have been connected by a Gigaethernet.

\section{Conclusions}

In the paper, the so-called structural solution has been applied to the solution of the elliptic partitial-differential equations. In the algorithm of the computations some properties of the structural solution have been exploited, namely the fact that the solution is composed of two parts, one of them fulfils exactly the boundary conditions and the others fulfils the differential equation in an approximate way. This feature of the solution can be employed effectively if we assume simple, linear basis functions over local simplexes and use the structured grid of nodes. That, together with the "row-by-row" method of computing the coefficients of the resulting system of linear algebraic equations, leads to the effective parallel algorithm of the solution.

In the authors' opinion, the efficiency of the proposed method should be particularly observable in the analysis of the problems with real great domain solutions. On the other hand, if more complex boundary value problems are to be solved, the local basis spline functions of the higher order will probably be needed.

\section{References}

1. Grzymkowski, R., Korek, K.: On R-function Theory and its Application in Inverse Problem of Heat Conduction. Information Technology Interfaces. In: Proceedings of the $23^{\text {rd }}$ International Conference on Pula, Croatia, pp. 393-402 (2001)

2. Reddy., J.N.: Applied Functional Analysis and Variational Methods in Engineering. McGraw-Hill Book Company, New York (1986)

3. Rvachew, W.L., Sliesarienko, A.P.: Algiebra łogiki i intierwalnyje prieobrazowanija w krajewych zadaczach (in Russian), Izd. Naukowa Dumka, Kijów (1976)

4. Shapiro, V.: Theory of R-functions and Applications, Technical Report, Cornell University (1988)

5. Wawrzynek., A.: Modelling of solidification and cooling of metals and heat diffusion problems by R-function method (in Polish), Zesz. Nauk. Pol Śląskiej, Mechanika 119, Gliwice, Poland (1994)

6. Yagawa, G.: Node-by-node parallel finite elements: a virtually meshless method. Int. J. Numer. Meth. Eng. 60(1), 69-102 (2004) 